\title{
Estudo retrospectivo dos casos diagnosticados como ameloblastoma no Serviço de Anatomia Patológica do Hospital Universitário Antônio Pedro entre 1997 e 2007
}

Primeira submissão em 04/06/08 Última submissão em 13/01/08 Aceito para publicação em 20/12/08 Publicado em 20/12/08

\section{A retrospective study of ameloblastoma in the Anatomic Pathology Service of Antônio Pedro University Hospital between 1997 and 2007}

Daniela Otero Pereira da Costa'; Marcelle Bairral Ecard²; Silvia Paula de Oliveira ${ }^{3}$; Licínio Esmeraldo da Silva4; Eliane Pedra Dias ${ }^{5}$; Simone de Queiroz Chaves Lourenço ${ }^{6}$

\section{unitermos Ameloblastoma \\ Tumor odontogênico \\ Tumores dos maxilares}

\section{resumo}

Introdução: O ameloblastoma é um tumor odontogênico de origem epitelial que, pela freqüência, subtipos clínicos, grande capacidade infiltrativa e alta tendência à recorrência tem merecido bastante atenção. Objetivo: Realizar estudo retrospectivo dos casos diagnosticados histopatologicamente de ameloblastoma no Serviço de Anatomia Patológica do Hospital Universitário Antônio Pedro da Universidade Federal Fluminense (HUAP/UFF) no período de 1997 a 2007. Material e métodos: Foram selecionados casos de ameloblastoma correspondentes ao período da pesquisa, e dados como idade, sexo, raça, localização da lesão, aspecto radiográfico, tipo de biópsia, diagnósticos clínico e histopatológico foram compilados das fichas de requisição e/ou prontuários. Resultados: Depois do levantamento e da revisão das lâminas para confirmação do diagnóstico de ameloblastoma, a amostra totalizada foi de 23 casos. Os pacientes mais acometidos eram do sexo masculino, leucodermas e estavam entre a primeira e a terceira década de vida. Todos os casos ocorreram na mandíbula, sendo a região posterior a mais afetada. Predominaram o aspecto radiográfico multilocular e, histopatologicamente, 0 padrão multicístico, sendo os subtipos folicular e híbrido os mais freqüentes. Não foram observados casos de ameloblastoma desmoplásico e de células basais. Conclusões: A freqüência do ameloblastoma varia de acordo com a região geográfica avaliada, e a realização de estudos retrospectivos pode contribuir para a identificação de grupos e dos possíveis fatores associados ao seu desenvolvimento.

\section{abstract}

Background: Ameloblastoma is an odontogenic tumor of epithelial origin that has received considerable attention due to its frequency, clinical subtypes and high tendency to infiltrate and recur. Objective: To perform a retrospective study of cases histopathologically diagnosed of ameloblastoma at the Pathological Anatomy Department of APUH/UFF-RI from 1997 to 2007. Material and methods: We selected cases of ameloblastoma corresponding to the period of this research. Data such as age, gender, race, location of lesion, radiographic features, type of biopsy, clinical diagnosis, and histopathological diagnosis were compiled from patients' records. Results: After the investigation and review of slides for confirmation of the diagnosis of ameloblastoma, the sample comprised 23 cases. Most patients with ameloblastoma were male, Caucasian and age range 10-30. All lesions were in the mandible, mainly in the posterior region. The multilocular radiographic types were predominant, and histopathologically, multicystic type, whereas the follicular and hybrid ones were more frequent. There were no cases of desmoplastic and basal cells ameloblastoma. Conclusions: The frequency of ameloblastoma varies according to geographic analyzed region, and retrospective studies can contribute to identify groups and possible factors associated with its development. key words

Ameloblastoma

Odontogenic tumors

Jaw tumours

1. Mestranda do Programa de Pós-Graduação em Patologia da Universidade Federal Fluminense (UFF).

2. Especialista em Estomatologia pela Odontoclínica Central do Exército (OCEx).

3. Doutoranda do Programa de Pós-Graduação em Patologia da UFF; coordenadora do Curso de Especialização em Estomatologia da OCEx.

4. Professor-adjunto do Departamento de Estatística da UFF.

5. Coordenadora do Programa de Pós-Graduação em Patologia da UFF.

6. Professora-adjunta do programa de Pós-Graduação em Patologia da UFF. 


\section{Introdução}

O ameloblastoma é o tumor odontogênico de origem epitelial mais comum, destacando-se também pela diversidade de subtipos clínicos, a grande capacidade infiltrativa e a alta tendência à recorrência ${ }^{(2,8,9,15,17,26,27)}$.

Diversas hipóteses foram formuladas para esclarecer a etiologia desse tumor que, no entanto, permanece incerta. Avanços na área de biologia molecular indicam que determinadas alterações nos genes reguladores da odontogênese, como o PTCH, podem levar à formação desses tumores ${ }^{(11)}$.

Segundo a literatura consultada, os ameloblastomas originam-se de remanescentes epiteliais de localização intra e/ou extra-óssea. Estes podem ser derivados do epitélio da lâmina dentária (restos de Serres), do epitélio reduzido do órgão do esmalte, de restos de Malassez e das células da camada basal do epitélio de mucosa ${ }^{(23)}$.

As características epidemiológicas do ameloblastoma retratam um tumor que afeta predominantemente pacientes na quarta e quinta décadas de vida ${ }^{(3,18,21,25)}$, sendo rara a sua observação em crianças ${ }^{(15)}$. Não há preferência por sexo ou raça ${ }^{(3,9,10,13,21,27)}$. A localização preferencial é a região posterior da mandíbula em aproximadamente $80 \%$ dos casos; apenas $20 \%$ afetam a maxila $(3,16,21,26)$.

Segundo Regezi e Scubba ${ }^{(21)}$, os ameloblastomas geralmente são assintomáticos, sendo descobertos durante exame radiográfico de rotina. Nos casos mais agressivos podem ocasionar sintomatologia e expansão dos maxilares. Os exames de imagem são importantes para auxiliar o diagnóstico dos ameloblastomas. Radiograficamente apresentam-se como imagens radiolúcidas uni ou multiloculares, com erosão óssea ou com leve opacidade ${ }^{(9,19)}$, podendo estar associados a dentes inclusos e causar reabsorção de raízes $^{(16)}$.

Os ameloblastomas podem ser multicísticos (sólidos), unicísticos, desmoplásicos e periféricos ou extra-ósseos. Esse último aspecto muitas vezes não apresenta imagem radiográfica ${ }^{(9,16)}$.

Em relação aos padrões histopatológicos, dividem-se em foliculares, plexiformes, acantomatosos, células granulares, células basais e desmoplásicos. No entanto, em alguns casos pode haver associação de variantes, sendo denominados de "híbridos"(9,16).

Trabalhos epidemiológicos referentes à incidência e à caracterização do ameloblastoma estão sendo desenvolvidos em diversos países ${ }^{(1,2,5,7,10,12-14,19,20,22, ~ 24-26, ~ 28) . ~}$
Chidzonga et $a l .{ }^{(5)}$ realizaram um estudo retrospectivo dos ameloblastomas no Hospital Universitário de Zimbábue (África) num período de 10 anos. O número total de lesões bucais foi de 1.723 , das quais $117(6,8 \%)$ foram ameloblastomas. Pacientes na segunda e terceira décadas de vida foram mais acometidos e houve discreta predileção por homens. Todas as lesões possuíram localização intra-óssea com considerável predomínio pela mandíbula $(95,7 \%)$, e a região posterior compreendida por molares, ângulo e ramo foi a mais acometida (34,8\%). O aspecto radiográfico multilocular predominou em $81,2 \%$ dos casos. O ameloblastoma unicístico e a variante folicular foram os mais freqüentes, representando $70,1 \%$ e $68,4 \%$, respectivamente.

O objetivo do nosso trabalho foi analisar os casos diagnosticados histopatologicamente como ameloblastoma no Serviço de Anatomia Patológica do HUAP/UFF no período de 1997 a 2007, adotando a classificação histopatológica da Organização Mundial da Saúde (OMS).

\section{Material e métodos}

Este trabalho, submetido e aprovado pelo comitê de ética em pesquisa da Faculdade de Medicina da UFF, sob O Protocolo no $167 / 08$, consistiu no levantamento retrospectivo de todos os casos com diagnóstico histopatológico de ameloblastoma, no período de 1997 a 2007, no Serviço de Anatomia Patológica do HUAP.

Os critérios de inclusão adotados foram casos diagnosticados de ameloblastoma no período de 1997 a 2007 com fichas clínicas ou prontuários; e os de exclusão foram indisponibilidade de blocos de parafina e/ou fichas e prontuários e lâminas com impossibilidade de diagnóstico.

Foram compilados das fichas de requisição de exames e/ou prontuários dados referentes ao paciente, como idade, sexo e raça, além daqueles referentes à lesão propriamente dita, como localização, aspectos radiográficos, tipo de biópsia, características clínicas e histopatológicas.

As lâminas coradas em hematoxilina e eosina (HE) dos casos diagnosticados como ameloblastoma foram reavaliadas histopatologicamente por dois observadores independentes e, quando necessário, foram reclassificadas de acordo com a classificação histopatológica proposta pela OMS (2005).

Todos os dados obtidos foram inseridos e tabulados utilizando-se o programa Microsoft Office Excel 2003, da Microsoft, e a descrição estatística foi realizada pelo programa SPSS versão 10.0 para Windows, da SPSS INC. 


\section{Resultados}

Foram 4.321 os resultados obtidos das lesões bucais encaminhadas para diagnóstico anatomopatológico no HUAP entre 1997 e 2007, sendo 126 casos de tumores odontogênicos, representando 2,91\%. Dessas neoplasias odontogênicas, 24 foram diagnosticadas como ameloblastoma, correspondendo a 19\% dos casos (Tabela 1). É válido mencionar que um caso de ameloblastoma foi excluído devido à indisponibilidade do bloco de parafina.

Ao se relacionarem os casos de ameloblastoma com o sexo dos pacientes, observou-se discreta prevalência pelo sexo masculino, com 13 casos (56,5\%), e 10 do sexo feminino $(43,5 \%)$.

A faixa etária mais acometida foi a compreendida entre a primeira e a terceira década de vida, com 10 casos $(43,5 \%)$, seguida da quarta à sexta década, com oito casos $(34,8 \%)$, e acima da sétima, com cinco casos (21,7\%). A idade dos pacientes variou de 10 a 82 anos, sendo a idade média 39,2 anos (desvio padrão [DP] = 19,3 anos).

Em relação à raça, sete $(30,4 \%)$ eram brancos, sete $(30,4 \%)$ negros, um pardo $(4,3 \%)$ e em oito $(34,8 \%)$ casos não havia essa informação nas fichas de requisição dos pacientes.

Todos os casos de ameloblastoma encontrados nesse estudo ocorreram na mandíbula, sendo três (13\%) com localização na região anterior, quatro $(17,4 \%)$ na média, um $(4,3 \%)$ envolvendo as regiões anterior e posterior e 14 (60,9\%) em região posterior. Em apenas um caso não havia essa informação.

Quanto ao aspecto radiográfico, somente um caso $(4,8 \%)$ era da variante periférica, não possuindo imagem radiográfica. Entre os casos analisados, houve predominância do aspecto multilocular com 10 casos (43,5\%), enquanto cinco casos $(21,7 \%)$ eram do tipo unilocular. Além disso, essa informação não foi obtida em oito casos (34,7\%).

Dos tipos clínicos descritos na literatura, o multicístico ou sólido foi o mais freqüente, com 18 casos (78,3\%), seguido do unicístico, com quatro casos $(17,4 \%)$, e, por último, o periférico, com apenas um caso (4,3\%). Nenhum caso da variante desmoplásica foi relatado.

Quanto ao padrão histopatológico do ameloblastoma multicístico, foram observados sete casos $(30,4 \%)$ foliculares, três (13\%) plexiformes e um $(4,8 \%)$ de células granulares. A combinação de mais de um padrão foi encontrada em sete casos, caracterizada pela variante folicular com áreas acantomatosas (quatro casos - 17,4\%), folicular com
Tabela 1 Perfil do ameloblastoma na amostra

\begin{tabular}{|c|c|c|}
\hline Fatores & $n$ & Estatística \\
\hline Pacientes & 23 & $100 \%$ \\
\hline \multicolumn{3}{|l|}{ Sexo } \\
\hline Feminino & 10 & $43,5 \%$ \\
\hline Masculino & 13 & $56,5 \%$ \\
\hline \multicolumn{3}{|l|}{ Idade } \\
\hline Média & & $\begin{array}{c}39,2 \text { anos } \\
(\mathrm{DP}=19,3 \text { anos })\end{array}$ \\
\hline Mínima & & 10 anos \\
\hline Máxima & & 82 anos \\
\hline \multicolumn{3}{|l|}{ Raça } \\
\hline Leucoderma & 7 & $30,4 \%$ \\
\hline Melanoderma & 7 & $30,4 \%$ \\
\hline Feoderma & 1 & $4,3 \%$ \\
\hline Sem informação & 8 & $34,8 \%$ \\
\hline \multicolumn{3}{|l|}{ Região da mandíbula } \\
\hline Anterior & 3 & $13 \%$ \\
\hline Média & 4 & $17,4 \%$ \\
\hline Posterior & 14 & $60,9 \%$ \\
\hline Ântero-posterior & 1 & $4,3 \%$ \\
\hline Sem informação & 1 & $4,3 \%$ \\
\hline \multicolumn{3}{|l|}{ Aspecto radiográfico } \\
\hline Unilocular & 5 & $21,7 \%$ \\
\hline Multilocular & 10 & $43,5 \%$ \\
\hline Sem informação & 8 & $34,7 \%$ \\
\hline \multicolumn{3}{|l|}{ Tipo de biópsia } \\
\hline Incisional & 9 & $39,1 \%$ \\
\hline Excisional & 10 & $43,5 \%$ \\
\hline Sem informação & 4 & $17,4 \%$ \\
\hline \multicolumn{3}{|l|}{ Diagnóstico clínico } \\
\hline Unicístico & 4 & $17,4 \%$ \\
\hline Sólido & 18 & $78,3 \%$ \\
\hline Periférico & 1 & $4,3 \%$ \\
\hline \multicolumn{3}{|c|}{ Diagnóstico histopatológico } \\
\hline Folicular & 7 & $30,4 \%$ \\
\hline Plexiforme & 3 & $13 \%$ \\
\hline $\begin{array}{l}\text { Folicular } \\
\text { com áreas } \\
\text { acantomatosas }\end{array}$ & 4 & $17,4 \%$ \\
\hline $\begin{array}{l}\text { Folicular } \\
\text { com áreas } \\
\text { plexiformes }\end{array}$ & 1 & $4,3 \%$ \\
\hline $\begin{array}{l}\text { Plexiforme com } \\
\text { áreas foliculares }\end{array}$ & 2 & $8,7 \%$ \\
\hline Luminal & 1 & $4,3 \%$ \\
\hline Mural & 2 & $8,7 \%$ \\
\hline $\begin{array}{l}\text { Células } \\
\text { granulares }\end{array}$ & 1 & $4,3 \%$ \\
\hline Sem informação & 2 & $8,7 \%$ \\
\hline
\end{tabular}


áreas plexiformes (um caso - 4,3\%) e plexiforme com áreas foliculares (dois casos - 8,7\%) (Figura 1).

Em relação às variantes do ameloblastoma unicístico, foram encontrados apenas dois casos $(8,7 \%)$ de proliferação mural e um caso $(4,3 \%)$ de proliferação ameloblástica luminal. No entanto, em dois casos $(8,7 \%)$, por se tratarem de biópsias incisionais, não foi possível aplicar a classificação histopatológica.

Ao avaliarmos o tipo de biópsia, a excisional foi a mais comum, estando presente em 10 casos (43,5\%); a incisional representou nove $(39,1 \%)$. No entanto é válido mencionar que, nas situações em que haviam sido realizados os dois procedimentos, apenas a biópsia excisional foi computada, fato que se deve à possibilidade de melhor análise do material a ser estudado. Em quatro casos não havia informação sobre o tipo de biópsia.

\section{Discussão}

O ameloblastoma é um tumor odontogênico de origem epitelial com localização intra-óssea e muitas vezes não apresenta sintomatologia, sendo, por isso, nessas ocasiões diagnosticado em estágios mais avançados ${ }^{(18)}$. Esse tumor acomete principalmente pacientes na quarta e quinta décadas de vida ${ }^{(3,18,21,27)}$. No entanto, nossos achados corroboram os de Chidzonga et al..$^{(5)}$ e Anjos et al.(2) de que os ameloblastomas são mais comuns entre a primeira e a terceira década de vida. Esse fato tem sido atribuído ao aumento da realização de radiografias panorâmicas em pacientes jovens, possibilitando a descoberta mais precoce dessas lesões.

Os pacientes acometidos pela variante unicística são mais jovens, com idade média de 25 anos $^{(13,16)}$. Achados semelhantes ao da literatura foram encontrados no estudo descrito.

O presente trabalho, o de Chidzonga et al. ${ }^{(6)}$ e o de Potdar ${ }^{(18)}$ evidenciaram ocorrência praticamente igual em relação ao sexo dos pacientes, porém com discreta tendência ao sexo masculino. Contrariamente, Anjos et al. (2) identificaram discreta predominância do sexo feminino. Santos et al. ${ }^{(22)}$ observaram prevalência mais acentuada do sexo feminino. Para Barbachan et al. ${ }^{(3)}$, Regezi e Scubba(21), Tommasi et al. ${ }^{(27)}$, Morales et al..(14),

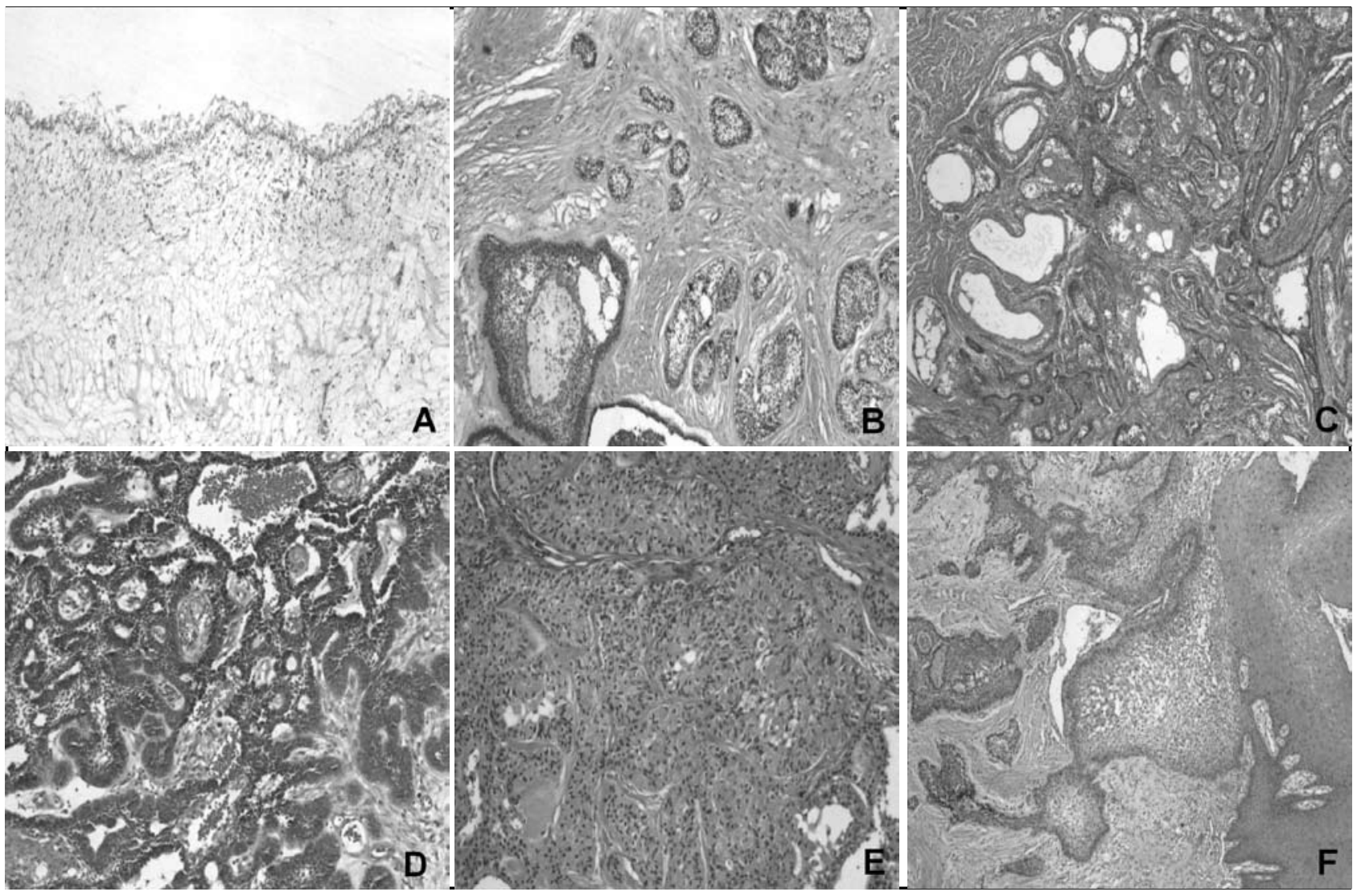

Figura 1 - Fotomicrografias dos padrões histopatológicos do ameloblastoma (HE, aumento inicial de 100x). A: unicístico; B: folicular; C: folicular exibindo áreas acantomatosas (hibrido); D: plexiforme; E: células granulares; F: periférico exibindo revestimento epitelial 
Montes et al. ${ }^{(13)}$ não houve diferença entre os sexos dos pacientes.

A alta freqüência de pacientes negros acometidos por ameloblastoma relatada no continente africano é discutida por Mosadomi et al. ${ }^{(12)}$. Os autores atribuíram esses achados ao processo de coleta da amostra, que se caracteriza por tumores maxilares benignos, de crescimento lento, assintomático e que não foram tratados ou investigados na África nas duas últimas décadas. Assim, os pacientes foram diagnosticados tardiamente e em grande número, tornando questionáveis os dados dos estudos. O trabalho atual encontrou $42,9 \%$ brancos; $33,3 \%$ negros; $4,3 \%$ pardos e em $23,8 \%$ dos casos não havia tal informação.

Ao avaliar o sítio anatômico de acometimento desse tumor, os autores são unânimes em afirmar que a localização preferencial é a mandíbula, principalmente a região posterior $^{(9,12,13,16)}$. O presente estudo não encontrou nenhum caso de tumor na maxila.

As características radiográficas dos ameloblastomas variam de imagem radiolúcida, com erosão óssea a uma leve radiopacidade ${ }^{(19)}$. Os achados nesse estudo corroboraram outros nos quais o aspecto mais comum é de uma lesão radiolúcida multilocular, que pode ser caracterizada por favos de mel ou bolhas de sabão(5, 12, 18). Outros autores descreveram também a presença de reabsorção de raízes ${ }^{(16)}$ e expansão óssea ${ }^{(16,18)}$ como achados radiográficos comuns. Em seu trabalho, Reichart et al. ${ }^{(19)}$ encontraram leve predominância da imagem unicística. A associação entre dente incluso e essa variante foi relatada com freqüência por Chidzonga et al. ${ }^{(5)}$ e Neville et al. ${ }^{(16)}$.

Os autores revistos na literatura ${ }^{(16,19,26)}$ apontaram o ameloblastoma multicístico ou sólido como o mais freqüente. No presente estudo $78,94 \%$ dos casos corresponderam a essa variante. Contrariamente, nos trabalhos de Chidzonga et al.(6), Morales et al.(14) e Montes et al.(13) foi observado que a variante unicística foi a mais usual. Além das variantes supracitadas, existem a periférica, representada por $4,3 \%$, e a desmoplásica, com nenhum caso. Os dados do nosso estudo concordam com a literatura quanto a que ambas as variantes são pouco freqüentes ${ }^{(1,12,13,22)}$.

Sobre os padrões histopatológicos presentes no ameloblastoma existe grande divergência na literatura. O presente estudo encontrou resultados idênticos entre o padrão folicular e o híbrido, ambos representados por $30,4 \%$ dos casos. Mosadomi et al.(12) encontraram associação entre as variantes folicular e plexiforme em aproximadamente 16\% dos casos. Segundo Adebiy ${ }^{(1)}$, Chidzonga et al. ${ }^{5}$, Reichart et al. ${ }^{(19)}$ e Neville et al. ${ }^{(16)}$, Simon et al. ${ }^{(23)}$ relataram que o padrão mais comum é o folicular. Autores como Anjos et al.(2) e Santos et al.(22) observaram o predomínio da variante plexiforme.

Estudos apontam a existência de correlação entre os subtipos histopatológicos do ameloblastoma com a agressividade e a taxa de recorrência do tumor ${ }^{(2,4,6,19)}$. Anjos et al.(2) e Reichart et al.(19) observaram maior tendência a recidiva nos casos das variantes folicular, plexiforme e acantomatosa. Deshpande et al. ${ }^{(6)}$ e Braunshtein et al. ${ }^{(4)}$ acreditavam que o padrão de células granulares e a presença de células claras caracterizavam o tumor como mais agressivo do que os outros subtipos, respectivamente. No entanto, para Reichart e Philipsen ${ }^{(20)}$ não há significância entre a variante histopatológica e o prognóstico ou a conduta clínica. Eles acrescentam ainda que a sugestão de maior agressividade das células granulares não é válida e que as células claras merecem grande atenção por poderem fazer parte de uma neoplasia maligna.

Embora diversos subtipos de ameloblastoma tenham sido encontrados, grande parte das biópsias realizadas foram incisionais. Assim, a avaliação do padrão histopatológico predominante pode ser questionada. O padrão predominante real do ameloblastoma só é definido após o envio da peça cirúrgica para a análise anatomopatológica.

Estudos sobre tumores odontogênicos de diferentes países apontam o ameloblastoma e o odontoma como as lesões odontogênicas mais comuns. De acordo com o país avaliado, existe grande discrepância na freqüência desses tumores. Na África ${ }^{(12)}$, China $^{(28)}$ e Tanzânia(23) há alta incidência de ameloblastomas, que variam de 50\% a $80 \%$ de todos os tumores odontogênicos. Entretanto, a freqüência dos odontomas nesses locais é de $2 \%$ a $7 \%$. Já em relação aos países das Américas ${ }^{(7,22,26)}$ e também na Europa, como na Estônia ${ }^{(25)}$, os resultados são contraditórios. Dessa forma, alguns autores acreditam que essa desigualdade é decorrente da variação geográfica. Para Simon et al. ${ }^{(24)}$ a alta freqüência dos ameloblastomas na África é decorrente principalmente da produção de sintomas incapacitantes que levam os pacientes a procurar tratamento. Os autores acrescentam ainda a falta de investigações mais acuradas e o diagnóstico tardio nos centros de atendimento. Outros afirmam que a incidência do odontoma em muitos países foi provavelmente subestimada pelas características clínicas da lesão de crescimento autolimitado, sem sintomatologia, sendo apenas diagnosticado por exames de imagem. 
Outra justificativa consiste na remoção desses tumores e no não-envio do material para exame e diagnóstico histopatológico(10).

\section{Conclusão}

Os resultados encontrados neste estudo permitem concluir que a freqüência do ameloblastoma é diferente em diversas regiões geográficas, além de algumas variáveis avaliadas na amostra, como predomínio do padrão histopatológico. Mediante esses tipos de estudo podem-se obter informações de grande valia a respeito dos aspectos clínico e histopatológico do ameloblastoma e, com isso, contribuir para a identificação dos grupos de risco e dos possíveis fatores associados ao seu desenvolvimento.

\section{Agradecimentos}

Os autores agradecem o apoio recebido pela Coordenação de Aperfeiçoamento de Pessoal de Nível Superior (CAPES) e ao Conselho Nacional de Desenvolvimento Científico e Tecnológico (CNPq).

\section{Referências}

1. ADEBIYI, K. E. et al. Clinicopathological analysis of histological variants of ameloblastoma in a suburban Nigerian population. Head \& Face Medicine, v. 2, n. 42, 2006. Disponível em: <http:// www.head-face med.com/content/2/1/42>. Acesso em: jul. 2007.

2. ANJOS, E. D. et al. Estudo clínico-patológico de ameloblastomas: análise de casos. Rev Bras Odontol, v. 60, n. 4, p. 224-8, 2003.

3. BARBACHAN, J. J. D. et al. Considerações sobre o estudo dos ameloblastomas. Rev Fac Odontol, v. 27, p. 13-25, 1985.

4. BRAUNSHTEIN, E. et al. Clear cell odontogenic carcinoma and clear cell ameloblastoma: a single clinicopathologic entity? A new case and comparative analysis of the literature. J Oral Maxillofac Surg, v. 61, n. 9, p. 1004-10, 2003

5. CHIDZONGA, M. M. et al. Ameloblastoma: the Zimbabwean experience over 10 years. Oral Surg Oral Med Oral Pathol Oral Radiol Endod, v. 82, p. 38- 41, 1996.

6. DESHPANDE, A. et al. Granular cell ameloblastoma of the jaw. A report of two cases with fine needle aspiration cytology. Acta Cytol, v. 44, n. 1, p. 81-5, 2000.

7. FERNANDES, A. M. et al. Odontogenic tumors: a study of 340 cases in a Brazilian population. J Oral Pathol Med, v. 34, p. 583-7, 2005.

8. GARDNER, D. G.; CORIO, R. L. The relationship of plexiform unicystic ameloblastoma to conventional ameloblastoma. Oral Surg, v. 56, p. 54-60, 1983.

9. GARDNER, D. G. et al. Ameloblastomas. In: BARES, L. et al. World Health Organization Classification of Tumours. Pathology and Genetic of Head and Neck Tumors. IARC Press: Lyon, 2005.

10. JING, W. et al. Odontogenic tumours: a retrospective study of 1642 cases in a Chinese population. Int J Oral Maxillofac Surg, v. 36, p. 20-5, 2007.

11. KAWABATA, T. et al. Polymorphisms in PTCH1 affect the risk of ameloblastoma. J Dent Res, v. 8, n. 9, p. 812-6, 2005.

12. MASODOMI, A. Odontogenic tumors in an African population: analysis of twenty-nine cases seen over a 5-year period. Oral Surg, v. 40, n. 4, p. 502-21, 1975.

13. MONTES, C. L. et al. Ameloblastomas:a regional LatinAmerican multicentric study. Oral Diseases, v. 13, p. 303-7, 2007.

14. MORALES, S. F. et al. Tumores odontogénicos en Puebla. Revista ADM, v. LVI, 5, p. 187-90, 1999.

15. MULLER, H; SLOOTWEG, P. J. The ameloblastoma, the controversial approach to therapy. J Max-fac Surg, v. 13, p. 79-87, 1985.

16. NEVILLE, B. W. et al. Patologia oral e maxilofacial. 2. ed. Rio de Janeiro: Guanabara Koogan, 2004.

17. OLALTAN, A. A. et al. Recurrent ameloblastoma of the jaws. A follow-up study. Int J Oral Maxillofac Surg, v. 27, p. $456-60,1998$

18. POTDAR, G. G. Ameloblastoma of the jaw as seen in Bombay, India. Oral Surg Oral Med Oral Pathol, v. 28, n. 3, p. 297-309, 1969.

19. REICHART, P. A. et al. Ameloblastoma: biological profile of 3,677 cases. Oral Oncol, Eur J Cancer, v. 31b, n. 2, p. 86-99, 1995. 
20. REICHART, P. A.; PHILIPSEN, H. P. Odontogenic tumors and allied lesions. 1. ed. London: Quintessence, 2004.

21. REGEZI, J. A.; SCUBBA, J. J. Patologia bucal, correlações clínico-patológicas. 1. ed. Rio de Janeiro: Guanabara Koogan, 1991.

22. SANTOS, J. N. et al. Odontogenic tumors: analysis of 127cases. Pesqui Odontol Bras, v. 15, n. 4, p. 308 $13,2001$.

23SAPP, J. P. et al. contemporary oral and maxillofacial pathology (Hardcover). 2. ed. Mosby, 2003.

24. SIMON, E. N. M. et al. A 4-year prospective study on epidemiology and clinicopathological presentation of odontogenic tumors in Tanzânia. Oral Surg Oral Med Oral Pathol Oral Radiol Oral Endod, v. 99, p. 598-602, 2005.
25. TAMME, T. Odontogenic tumours, a collaborative retrospective study of 75 cases covering more than 25 years from Estonia. J Craniomaxillofac Surg, v. 32, p. 161-5, 2004.

26. TAYLOR, A. M. et al. Odontogenic tumors in Mexico: a collaborative retrospective study of 349 cases. Oral Surg Oral Med Oral Pathol Oral Radiol Endod, v. 84, p. 84: 672-5, 1997.

27. TOMMASI, A. F. Diagnóstico em patologia bucal. 2. ed. São Paulo: Pancast Editorial, 1998.

28. YONG, L. et al. Odontogenic tumors: a demographic study of 759 cases in a Chinese population. Oral Surg Oral Med Oral Pathol Oral Radiol Oral Endod, v. 86, n. 6, p. $707-14,1998$. 